\title{
ON HARDY TYPE INEQUALITIES FOR WEIGHTED QUASIDEVIATION MEANS
}

\author{
Zsolt PÁles and PaweŁ PasteczKa
}

Abstract. Using recent results concerning the homogenization and the Hardy property of weighted means, we establish sharp Hardy constants for concave and monotone weighted quasideviation means and for a few particular subclasses of this broad family. More precisely, for a mean $\mathscr{D}$ like above and a sequence $\left(\lambda_{n}\right)$ of positive weights such that $\lambda_{n} /\left(\lambda_{1}+\ldots+\lambda_{n}\right)$ is nondecreasing, we determine the smallest number $H \in(1,+\infty]$ such that

$$
\sum_{n=1}^{\infty} \lambda_{n} \mathscr{D}\left(\left(x_{1}, \ldots, x_{n}\right),\left(\lambda_{1}, \ldots, \lambda_{n}\right)\right) \leqslant H \cdot \sum_{n=1}^{\infty} \lambda_{n} x_{n} \text { for all } x \in \ell_{1}(\lambda) .
$$

It turns out that $H$ depends only on the limit of the sequence $\left(\lambda_{n} /\left(\lambda_{1}+\ldots+\lambda_{n}\right)\right)$ and the behaviour of the mean $\mathscr{D}$ near zero.

Mathematics subject classification (2010): 26D15, 26E60, 39B62, 40D25.

Keywords and phrases: Weighted mean, Hardy inequality, Hardy constant, quasiarithmetic mean, quasideviation mean, Jensen concavity.

\section{REFERENCES}

[1] M. BAJRAKTAREviĆ, Sur une équation fonctionnelle aux valeurs moyennes, Glasnik Mat.-Fiz. Astronom. Društvo Mat. Fiz. Hrvatske Ser. II, 13:243-248, 1958.

[2] F. Bernstein And G. Doetsch, Zur Theorie der konvexen Funktionen, Math. Ann., 76(4):514 $526,1915$.

[3] T. Carleman, Sur les fonctions quasi-analitiques, Conférences faites au cinquième congrès des mathématiciens scandinaves, Helsinki, page 181-196, 1932.

[4] E. T. Copson, Note on Series of Positive Terms, J. London Math. Soc., s1-2(1):9-12, 1927.

[5] Z. DARócZY, A general inequality for means, Aequationes Math., 7(1):16-21, 1971.

[6] Z. DARóczy, Über eine Klasse von Mittelwerten, Publ. Math. Debrecen, 19:211-217 (1973), 1972.

[7] Z. Daróczy And L. LosoncZI, Über den Vergleich von Mittelwerten, Publ. Math. Debrecen, 17:289-297 (1971), 1970.

[8] Z. DarócZy And Zs. PÁles, On comparison of mean values, Publ. Math. Debrecen, 29(1-2):107$115,1982$.

[9] Z. DARóCZY AND Zs. PÁLEs, Multiplicative mean values and entropies, Functions, series, operators, Vol. I, II (Budapest, 1980), page 343-359. North-Holland, Amsterdam, 1983.

[10] J. Duncan And C. M. MCGregor, Carleman's Inequality, Amer. Math. Monthly, 110(5):424-431, 2003.

[11] E. B. ELLIOTT, A simple exposition of some recently proved facts as to convergency, J. London Math. Soc., 1:93-96, 1926.

[12] C. Gini, Di una formula compressiva delle medie, Metron, 13:3-22, 1938.

[13] G. H. HARDY, Note on a theorem of Hilbert, Math. Z., 6:314-317, 1920.

[14] G. H. Hardy, J. E. Littlewood, And G. Pólya, Inequalities, Cambridge University Press, Cambridge, 1934. (first edition), 1952 (second edition).

[15] K. KNOPP, Über Reihen mit positiven Gliedern, J. London Math. Soc., 3:205-211, 1928.

[16] A. Kufner, L. Maligranda, And L.-E. Persson, The Hardy Inequality: About Its History and Some Related Results, Vydavatelskỳ servis, 2007. 
[17] E. LANDAU, A note on a theorem concerning series of positive terms, J. London Math. Soc., 1:38-39, 1921.

[18] L. LosoncZI, Über den Vergleich von Mittelwerten die mit Gewichtsfunktionen gebildet sind, Publ. Math. Debrecen, 17:203-208 (1971), 1970.

[19] L. LosonczI, Subadditive Mittelwerte, Arch. Math. (Basel), 22:168-174, 1971.

[20] L. LosoncZI, Subhomogene Mittelwerte, Acta Math. Acad. Sci. Hungar., 22:187-195, 1971.

[21] L. LosoncZI, Über eine neue Klasse von Mittelwerten, Acta Sci. Math. (Szeged), 32:71-81, 1971.

[22] L. LosonczI, General inequalities for nonsymmetric means, Aequationes Math., 9:221-235, 1973.

[23] L. LosonczI, Inequalities for integral mean values, J. Math. Anal. Appl., 61(3):586-606, 1977.

[24] P. Mulholland, On the generalization of Hardy's inequality, J. London Math. Soc., 7:208-214, 1932.

[25] P. PAsteczKa, When is a family of generalized means a scale?, Real Anal. Exchange, 38(1):193-209, 2012/13.

[26] J. E. PeČARIĆ And K. B. Stolarsky, Carleman's inequality: history and new generalizations, Aequationes Math., 61(1-2):49-62, 2001.

[27] Zs. PÁLES, Characterization of quasideviation means, Acta Math. Acad. Sci. Hungar., 40(3-4):243260, 1982.

[28] Zs. PÁLES, On complementary inequalities, Publ. Math. Debrecen, 30(1-2):75-88, 1983.

[29] Zs. PÁLES, Inequalities for comparison of means, In W. Walter, editor, General Inequalities, 4 (Oberwolfach, 1983), volume 71 of International Series of Numerical Mathematics, page 59-73. Birkhäuser, Basel, 1984.

[30] Zs. PÁLES, Ingham Jessen's inequality for deviation means, Acta Sci. Math. (Szeged), 49(1-4):131$142,1985$.

[31] Zs. PÁLES, General inequalities for quasideviation means, Aequationes Math., 36(1):32-56, 1988.

[32] Zs. PÁLEs, On a Pexider-type functional equation for quasideviation means, Acta Math. Hungar., 51(1-2):205-224, 1988.

[33] Zs. PÁLEs, On homogeneous quasideviation means, Aequationes Math., 36(2-3):132-152, 1988.

[34] Zs. PÁLES, A Hahn-Banach theorem for separation of semigroups and its applications, Aequationes Math., 37(2-3):141-161, 1989.

[35] Zs. PÁles AND P. PASTECZKA, On the homogenization of means, Acta Math. Hungar. 159(2):537$562,2019$.

[36] Zs. PÁLES AND P. PASTECZKA, Characterization of the Hardy property of means and the best Hardy constants, Math. Ineq. Appl., 19:1141-1158, 2016.

[37] Zs. PÁLES AND P. PASTECZKA, On Kedlaya type inequalities for weighted means, J. Inequal. Appl., 2018(99), 2018.

[38] Zs. PÁles AND P. PAsteCZKa, On the best Hardy constant for quasi-arithmetic means and homogeneous deviation means, Math. Inequal. Appl., 21:585-599, 2018.

[39] Zs. PÁles And P. PAsteczKa, On Hardy type inequalities for weighted means, Banach J. Math. Anal., 13:217-233, 2019. 